\title{
Herpes zoster ophthalmicus: a medical review
}

\author{
SUSAN LIGHTMAN, R. J. MARSH, AND DAVID POWELL \\ From the Department of Clinical Ophthalmology, Moorfields Eye Hospital, City Road, London EC1V 2PD
}

SUMmaRY Patients with herpes zoster undergo extensive screening to detect underlying malignant disease which is compromising their immunity. In a restrospective survey of 1000 patients with herpes zoster ophthalmicus 12 patients had malignant disease which was known on presentation. No new cases were detected or discovered on follow-up. Three patients developed a disseminated rash, but none of these had an underlying malignant disease.

For many years it has been suggested that patients contracting herpes zoster have compromised imunity ${ }^{1-4}$ and the zoster infection is symptomatic of an underlying immunosuppressive process. However, zoster occurs widely in the community, especially in the elderly, and it was our impression that most of the patients we saw with herpes zoster ophthalmicus (which constitutes $7 \%$ of all zoster infection $)^{5}$ had good general health prior to contracting it.

We therefore decided to carry out a retrospective study on our patients to see if this impression was correct and to see if the infection and ocular complications were more severe in those patients with underlying disease.

\section{Patients and methods}

Our study was based on patients attending the Zoster Clinic at Moorfields Eye Hospital over the past 9 years. The vast majority of the patients were referred directly to the Casualty Department from their general practitioner within the first 10 days of onset of the rash. The diagnosis was confirmed in the Casualty Department and preliminary treatment started. Within the next few days the patients were seen in the Zoster Clinic, which is part of the External Diseases Group of Clinics under the auspices of the Professorial Unit at Moorfields. Apart from the ocular examination all patients had a medical history taken and underwent the following tests: a full blood count, differential white blood count and film, erythrocyte sedimentation rate (ESR), liver function tests, electrophoretic strip, blood sugar, and chest $x$-ray. The patients were also

Correspondence to Dr S. Lightman. asked about previous zoster infections and whether they were on systemic steroids. Regular follow-up appointments were then made until the eye was quiet or they defaulted.

Those patients whose blood tests were abnormal (abnormal being taken as anything outside the normal range in biochemistry, and differing from a normal differential white blood count) were looked at in detail and multivariate analysis was undertaken with age, sex, haematological results, biochemical results, and type of eye problem as variants. Probabilities were then computed by means of the Fisher exact probability test.

\section{Results}

One thousand patients have attended the Zoster Clinic in the last 9 years. A small proportion of the patients had no ocular involvement-the infection being confined to the skin. Thirty-six patients reported previous zoster infections. These had usually been thoracic or lumbar, but 1 patient had suffered ophthalmic zoster on the other side 18 years previously and had visible pitting and depigmentation in the skin on that side. Three patients developed a disseminated rash-with more than 5 spots in places other than those which could have been produced by auto-inoculation. None of these 3 patients had malignant disease or developed it during the period of follow-up.

Five patients were on steroids for unrelated problems including chronic chest disease, eczema, and nephrotic syndrome. Assuciated diseases included 4 with diabetes, 3 postgastrectomy patients (benign gastric ulcers), 1 patient with discoid lupus erythematosus, 1 with sarcoid, and 3 with ulcerative colitis. None of these were on steroids or had been 
for at least 4 months. Two patients developed transient hemiplegias on the opposite side to their zoster ophthalmicus. One episode was 6 weeks after the onset of the zoster and the other 5 months.

No previously undiagnosed malignancies were found or developed during the period of follow-up. Six patients had carcinoma of the breast, diagnosed between one and 21 years previously. Three had carcinoma of the colon; 1 patient was on chemotherapy and the other 2 had been diagnosed 2 and 6 years previously. There was 1 patient with Hodgkin's disease on chemotherapy, 1 with carcinoma of the lung diagnosed 10 years before, and 1 with a 'brain tumour' diagnosed in 1952.

Of the 1000 patients screened 364 had abnormal blood results of any sort, of which 72 patients had no ocular involvement. Table 1 shows the haematology results in this group. 176 were male and 188 were female. The commonest age range was 5070 years, with the youngest being 18 years and the eldest 91. The average period of follow-up was 2 years with the range being less than 1 year to 8 years. Most patients were followed up until their eye was quiet and they were discharged, with a very small proportion defaulting before this time.

The commonest eye problems which developed were iritis/iris atrophy, episcleritis, and superficial stromal scarring. The age and sex of the patient were irrelevant to the type of eye problem which developed, and there were no correlations with abnormal biochemical results. There were no prognostic indicators in the blood results. The outcome of the ocular disease was not affected by the presence or absence of a lymphocytosis, raised gamma globulins or any other blood factors. There ,was a significant association between the presence of an abnormal haematological result (as opposed to a normal result) and mucus plaque keratitis $(p<0.0005)$, but when the abnormal results were looked at no specific abnormality was more common.

When the different eye problems in the 364 patients were analysed, several significant associates emerged. Patients with iritis/iris atrophy had a higher incidence of keratitis $(p<0.001)$ and episcleritis $(p<0.01)$ than did the others. Similarly patients

Table 1 Haematology results in 364 patients

\begin{tabular}{lr}
\hline Result & No. of patients \\
\hline Normal & 225 \\
Lymphocytosis & 64 \\
Neutrophilia & 19 \\
Monocytosis & 19 \\
Leucopenia & 14 \\
Lymphopenia & 13 \\
Eosinophilia & 10 \\
\hline
\end{tabular}

with mucus plaque keratitis had a higher incidence of neuroparalytic ulcer $(p<0.01)$ and cataract $(p<0.001)$.

\section{Discussion}

Only 12 of our patients, who were from a general rather than a hospital population, had malignant disease, and no new malignancies were discovered on screening or developed during the follow-up period. This confirmed our clinical impression that we are dealing with a predominantly healthy population. Dissemination of the rash occurred in only 3 patients, and none of these had malignant disease. A previous study of 175 patients with herpes zoster had 17 patients who developed widespread vesicular lesions during the course of typical localised herpes zoster. Eleven of these 17 patients had serious underlying diseases. ${ }^{6}$ The difference in our results probably reflects the selection of patients, ours coming from the general population not from a hospital population.

Second attacks of zoster are thought to be rare. Head and Campbell found 4 patients in a survey of 400 patients, ${ }^{7}$ whereas 36 of our patients had suffered previous attacks. None of these patients had underlying disease nor a history suggestive of reinfection, so it is difficult to explain how they developed the disease.

The associates of eye complications are interesting. Both episcleritis and iritis involve an occlusive vasculitis and hence would be expected to be associated. Mucus plaque keratitis tends to be a chronic diffuse keratitis with progressive loss of corneal sensation, so it is not surprising that neuroparalytic ulceration occurs more commonly in this group. The high incidence of cataract in this group poses a problem in aetiology, since severe anterior segment ischaemia can lead to cataracts, as can intensive local steroid therapy.

This study has shown that the great majority of patients contracting herpes zoster ophthalmicus are healthy and therefore do not have diminished immunity. The trigger factors for this disease remain a puzzle and require further investigation.

Susan Lightman is supported by a grant from the Wellcome Trust.

\section{References}

1 Edgerton AE. Herpes zoster ophthalmicus. Arch Ophthalmol 1945; 34: 40-62.

2 Walsh FB, Hoyt WF. Clinical Neuro-Ophthalmology. 3rd Ed. Baltimore: Williams and Wilkins, 1969: 2: 1351-2. 
3 Wright ET, Winer LH. Herpes zoster and malignancy. Arch Dermatol 1961 ; 84: 242-4.

4 Sokal JE, Firat D. Varicella-zoster infection in Hodgkin's disease. Am J Med 1965; 39: 452-63.

5 Archard C. Zona ophthalmique. Paris Med 1924; 1: 285 (cited by Edgerton ${ }^{1}$ ).
6 Merselis JG jnr, Kaye D, Hook EW. Disseminated herpes zoster. Arch Intern Med 1964; 113: 679-86.

7 Head H, Campbell AW. Pathology of herpes zoster and its bearing on sensory localization. Brain 1900; 23: 353523. 